\title{
The language of possibility and probability: Effects of negation on meaning
}

\author{
VALERIE F. REYNA \\ Rockefeller University, New York, New York 10021
}

\begin{abstract}
Modal adjectives (e.g., "possible" and "probable") have been the subject of much discussion by linguists and logicians. For ordinary speakers, it was found that an important aspect of the meaning of modal adjectives is that they can all be used to qualify the truth of a statement: Subjects sorted modal adjectives according to similarity of meaning and then ordered the same adjectives solely according to their degree of qualification. The sorting data yielded a one-dimensional scaling solution of low stress that correlated highly with the results from the ordering task. Using the same techniques, negation was found to translate a modal adjective down the scale of qualification (e.g., "improbable" is more qualifying than "probable") so that the order of affirmative adjectives with respect to each other is preserved for the corresponding negated adjectives. Negation in this domain is more analogous to a subtractive, rather than multiplicative, process. Also, affixal negation, as in "improbable," was consistently found to be more qualifying than lexical negation, as in "not probable."
\end{abstract}

Many semantic domains contain words that are not explicitly ordered within their domains (e.g., kinship words). The relations among word meanings in such domains has often been described in terms of discrete contrasts (Leech, 1974; Lyons, 1977). For example, the discrete contrast of gender, male vs. female, distinguishes the meanings of the words "father," "brother," and "son" from the meanings of "mother," "sister," and “daughter," respectively (Clark \& Clark, 1977). Semantic theories have most commonly dealt with the representation of such discrete meaning contrasts (Holyoak \& Walker, 1976).

Discrete contrasts, however, have not proved adequate in characterizing word meanings that refer to intrinsically ordered concepts (Cliff, 1959; Holyoak \& Glass, 1978; Holyoak \& Walker, 1976). For example, Holyoak and Glass found that quantifiers (e.g., "all" and "some") appear to be represented in memory as continuous, rather than discrete, values along an internal quantity scale. Holyoak and Glass point out that "[continuous] magnitude information must be incorporated into any psychological theory of meaning," particularly for "the semantic representation of 'logical' words such as quantifiers" (1978, p. 262).

In this study, I examine the semantic structure underlying a class of "logical" words that appear to be similar in some respects to quantifiers: such adjectives as "possible" and "necessary." This class of adjectival

This research was supported in part by Grant BivS 77-11612 from the National Science Foundation to Rockfeller University. Correspondence concerning this article should be sent to Valerie F. Reyna, University of Texas at Dallas, Box 688, Department of Psychology, GR 4.1, Richardson, Texas 75080. thank George A. Miller for his support and assistance. operators has been the subject of much discussion by logicians and philosophers; the branch of logic devoted to their study is called modal logic (Hughes \& Cresswell, 1974). In modal logic, "possible" and "necessary" receive special, technical, interpretations. Consider the following statements, and assume that both are true: (1) All bachelors are unmarried. (2) No body travels faster than light. The first statement expresses a logical necessity, and logicians would call this statement a necessary truth: The statement is true "by definition," and no evidence is needed to support it. Although the second statement might be strongly supported by scientific evidence, it would not be described as logically necessary. The second statement would be considered logically possible, since it is neither inherently necessary nor impossible. Modal logic, then, provides one account, a technical account, of the meanings of "possible" and "necessary."

The technical and nontechnical interpretations of modal adjectives are presumably related, but the extent of their overlap is unclear. Similarly, quantifiers receive technical interpretations in formal logic as well as nontechnical interpretations in ordinary discourse. Technical and nontechnical interpretations for some quantifiers, however, have been found to differ. For example, the logical interpretation of "some" is "at least one, and possibly all," whereas the ordinary interpretation of "some" is "few, but not all" (Revlis, 1975). Since technical and nontechnical interpretations for logical words sometimes fail to correspond (Falmagne, 1975; Taplin \& Staudenmayer, 1973), logical interpretations for modal adjectives may not fully characterize their natural language interpretations.

In characterizing the linguistic, as opposed to logical, properties of modal words, Lyons (1977) argues that modals express degrees of qualification: Lyons accepts 
as modal any word used to qualify the truth of a statement. For example, a speaker can choose to make an unqualified statement such as "It will rain," or the speaker might qualify the truth of "It will rain" by saying "It is possible that it will rain." According to Lyons, a speaker can choose among many modal words in order to convey different degrees of qualification, including probabilistic adjectives (e.g., "likely").

Lyons' (1977) linguistic analysis of modals suggests that their psychological representation might be similar to that of quantifiers. Holyoak and Glass' (1978) results indicate that quantifiers are mentally represented as continuous magnitude values along a unidimensional scale, a scale reflecting degrees of quantity. Modal adjectives might be similarly represented along a unidimensional scale of degrees of qualification. Modal adjectives would be ordered along the scale according to the degree to which they qualified a statement's truth. One goal of this study is to examine the semantic dimensions that underlie the meanings of modal adjectives.

Modal adjectives resemble quantifiers in another respect: Negation enjoys a similar, special role in both domains. The two basic quantifiers, "all" and "some," can be interdefined using negation. For example, the sentence "Some of the nurses do not like doctors" is semantically equivalent to "Not all of the nurses like doctors." The two basic modal adjectives, "possible" and "necessary," can also be interdefined using negation: The sentence "It is possible that the nurses do not like doctors" is semantically equivalent to "It is not necessary that the nurses like doctors." Another goal of this study is to examine the effects of negation on semantic relations among modal adjectives.

The presence of a negative component has commonly been used to account for the meaning relations between words, as in "happy-unhappy," "button-unbutton," and "same-different" (Clark \& Clark, 1977; Miller \& Johnson-Laird, 1976). The effect of negation on relations among words varies, however, from one domain to another. Consider the adjectives "reliable" and "trustworthy," shown in Figure 1. According to Funk and Wagnall's (1977) Standard College Dictionary, "trustworthy" is stronger than "reliable," implying that "our knowledge is complete and profound": Along the dimension of trustworthiness, "trustworthy" is more positive than "reliable." Negating these adjectives by prefixing them with "un" inverts their order along the dimension of trustworthiness: "Unreliable" is more positive than "untrustworthy." On the other hand, consider the adjectives "enjoyable" and "tolerable" (Figure 1). "Enjoyable" is stronger than "tolerable": Along the dimension of enjoyableness, "enjoyable" is more positive than "tolerable." Prefixing these adjectives with "un," however, preserves their order: "Unenjoyable" is more positive than "intolerable." Negated adjectives express lower values along a dimension (e.g.,

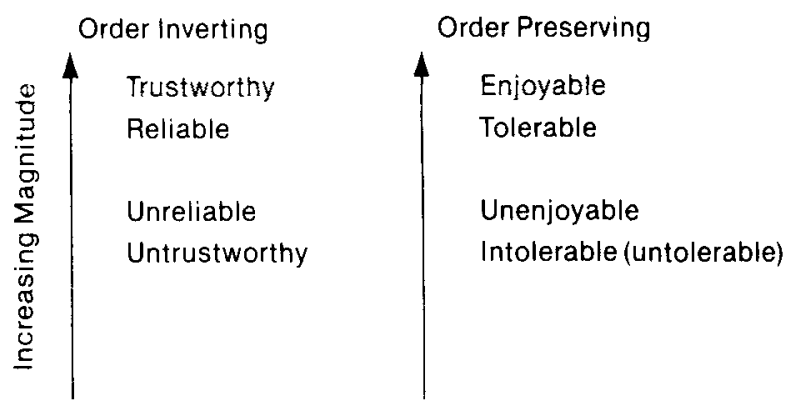

Figure 1. The effect of negation on relations among words in different semantic domains.

less enjoyableness), but in some cases their order along a dimension is preserved and in others their order is inverted.

English provides at least two options for negation: affixal negation, as in "impossible" and "unnecessary," and lexical negation, as in "not possible" and "not necessary." According to Zimmer (1964), affixal negation is interpreted as strong negation, whereas negation with "not" is interpreted as either strong or weak negation. In other words, "John is not happy" can be interpreted strongly as "John is unhappy" or weakly, as in "John is not happy, but he's not sad." Also, psychological research has demonstrated that sentences containing negation with "not" take longer to verify than sentences containing implicit negatives (Clark, 1972). Since available evidence suggests that these two types of negation are not equivalent, both types were used in the present study.

The initial problem is to determine the semantic dimensions that underlie the meanings of modal adjectives. The adjectives included in this study were "possible" and "necessary" and six other adjectives that seem to satisfy Lyons' (1977) definition of a modal word: "certain," "definite," "probable," "likely," "feasible," and "conceivable." Multidimensional scaling techniques have been used effectively to determine the semantic dimensions that underlie the meanings of lexical items (Miller, 1971). One procedure is to ask people to sort words into groups on the basis of similarity of meaning. The assumption is that the more similar in meaning two words are, the more often people will agree in grouping them together. Similarity is represented in terms of distance in space by a multidimensional scaling program (Young \& Torgerson, 1967): The more similar the items are, the closer they are in space. A multidimensional scaling program usually produces alternative solutions in spaces of one dimension, two dimensions, and so on, and determines how well the observed data are fit by solutions of each dimensionality. Once items are represented in a space, the dimensions along which items vary can be interpreted. This sorting and scaling method was used in the present study; if one semantic dimension, a dimension of 
qualification, adequately characterizes the meaning of modal adjectives, a one-dimensional solution should be obtained that fits the observed data well.

To establish a more precise interpretation for this dimension and to evaluate the hypothesis that this dimension reflects degrees of qualification, an additional task was administered: People ordered modal adjectives explicitly according to their degree of qualification. If qualification is an important semantic dimension for modal adjectives, this ordering according to degree of qualification should match the order of modal adjectives according to the one-dimensional solution. In other words, ordering modal adjectives simply on the basis of their degree of qualification should correspond to sorting them on the basis of similarity of meaning.

\section{EXPERIMENT 1}

\section{Method}

Subjects. Thirty-four adult volunteers were paid $\$ 3 / \mathrm{h}$ for their participation in the experiment. Half of the subjects participated in the affixal negation condition first, and the other half participated in the lexical negation condition first.

Materials. The stimuli were sentences of the form "It is that it will happen." In the affixal negation condition, eight affirmative modal adjectives and their eight affixal negations were substituted into this frame. The eight affirmative modal adjectives were "definite," "certain," "necessary," "probable," "likely," "possible," "feasible," and "conceivable," and their eight affixal negations were "indefinite," "uncertain," "unnecessary," "improbable," "unlikely," "impossible," "unfeasible," and "inconceivable." In the lexical negation condition, the same eight affirmative modal adjectives and their lexical negations were substituted into this frame. The affirmative adjectives were lexically negated by inserting "not" in front of each affirmative adjective. Thus, in each condition, 16 modal sentences were presented that were identical except for the modal adjective. Each sentence was typed on a separate $4 \times 6$ in. card. The affixal negation and lexical negation conditions differed only in the type of negation used.

Procedure. The order of participation in the affixal negation and the lexical negation conditions was counterbalanced across subjects. Each condition consisted of two tasks, a sorting task and an ordering task. Within each condition, subjects received the same 16 sentences in both tasks. The sorting task always preceded the ordering task. For each task, the stimuli were presented in a different random order for each subject.

Sorting task. Subjects sorted 16 sentences into groups such that all sentences within any group were judged to be similar in meaning. No constraints were placed on either the number of groups a subject was allowed to use or the number of sentences placed in any group.

Ordering task. Subjects ordered the modal adjectives according to their degree of qualification (i.e., subjects ordered the adjectives according to the degree to which each adjective qualified the statement "it will happen"). Qualifying the occurrence of an event seems to be equivalent to describing the event as more or less certain. Thus, subjects were instructed to put the 16 sentences in order according to the certainty of the event from the "least certain that it will happen" to the "most certain that it will happen."

\section{Results}

The sorting tasks in the affixal negation and lexical negation conditions each yielded a 16 by 16 similarity matrix: The entry in any cell was the number of subjects who judged the row word of the cell similar to the column word of that cell. These data were analyzed by the TORSCA multidimensional scaling program (Young \& Torgerson, 1967). There was no effect of order of administration for either the sorting or the ordering task. Therefore, the results presented below are for data collapsed across order of administration.

Results for the affixal negation condition will be presented first. The sorting data from the affixal negation condition yielded a one-dimensional solution of low stress (stress $=.0281$ ). The one-dimensional solution is presented on the left in Figure 2. The modal adjectives range from "certain" or "definite" at the top to "impossible" at the bottom.

The ordering data were analyzed by assigning a position from 1 to 16 to each sentence based on a subject's ordering of the sentences. Figure 2 shows the median position assigned to each sentence. Based on their median positions, the modal adjectives are arranged from the least qualifying, "certain," at the top, to the most qualifying, "impossible," at the bottom. The largest interquartile range was 3.06 , but the range usually did not exceed 2.00 .

The order of the modal adjectives based on the onedimensional solution corresponds to the order of the

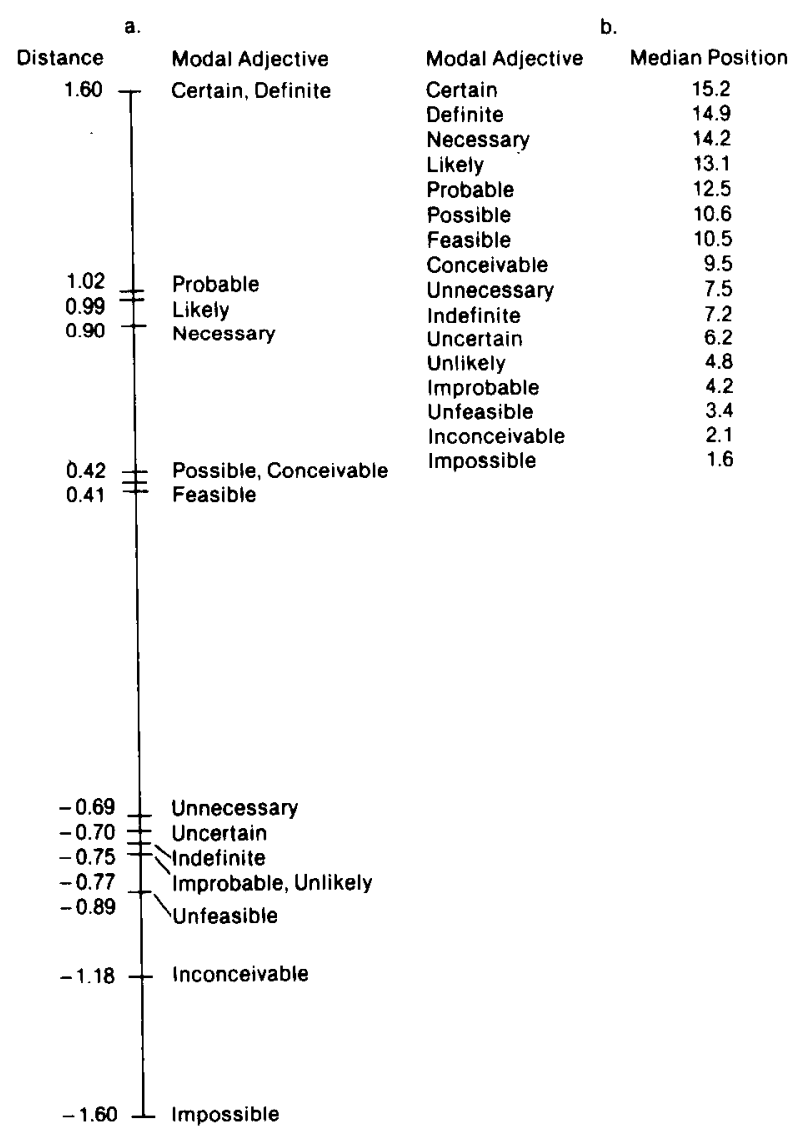

Figure 2. Affixal negation condition, Experiment 1: (a) Onedimensional scaling solution based on sorting modal adjectives according to similarity of meaning. (b) Ordering of modal adjectives according to their degree of qualification. 
adjectives based on the ordering results. The Spearman rank-order correlation for these two results is .98 $(\mathrm{p}<.001)$. In other words, in the affixal negation condition, the sorting data yielded a one-dimensional scaling solution of low stress that correlated highly with ordering modal adjectives according to their degree of qualification.

The one-dimensional solution obtained in the lexical negation condition is shown on the left in Figure 3. The one-dimensional solution fit the data well, but the stress was somewhat greater than that obtained in the affixal negation condition (stress $=.1156$ ). The ordering results from the lexical negation condition are presented on the right in Figure 3. The largest interquartile range was 3.45 , but the range usually did not exceed 2.50 . Again, the order of the adjectives based on the onedimensional solution correlated highly with their order based on the ordering results $\left(r_{s}=.94, p<.001\right)$; sorting the adjectives according to similarity of meaning corresponded to ordering them according to their degree of qualification.

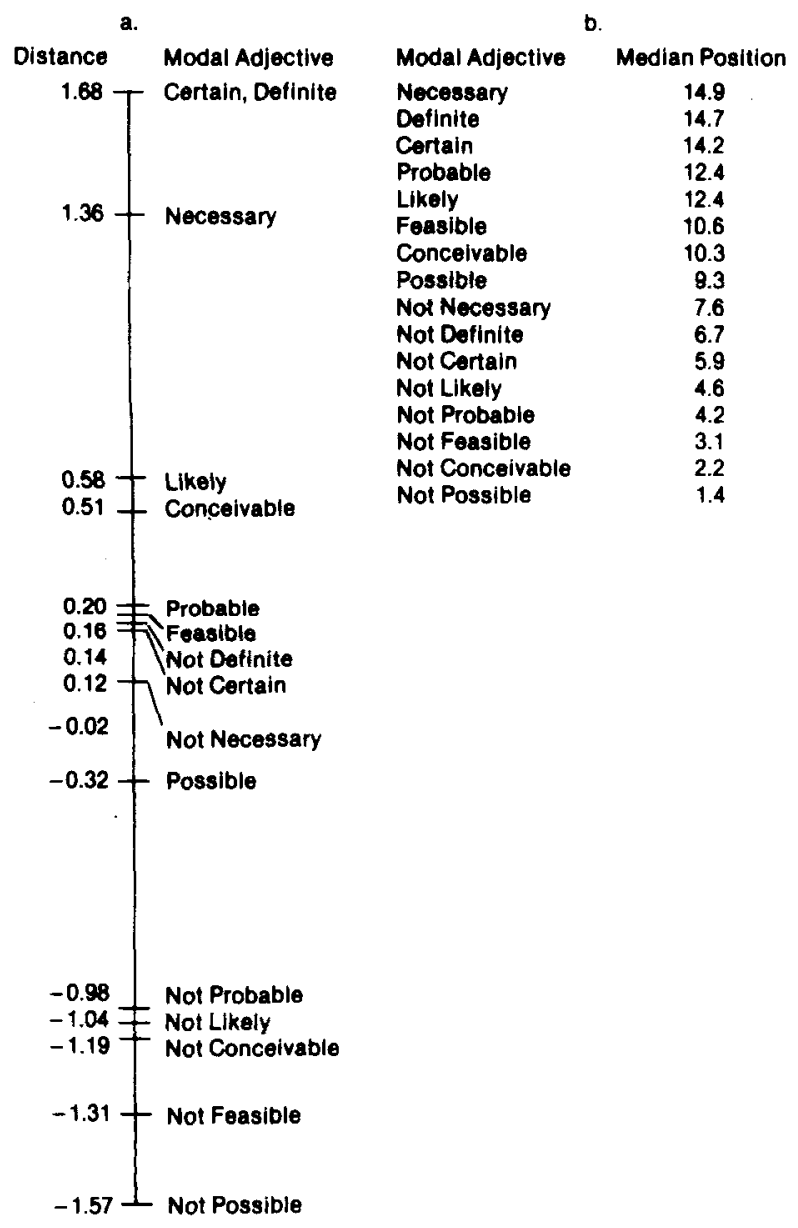

Figure 3. Lexical negation condition, Experiment 1 : (a) Onedimensional scaling solution based on sorting modal adjectives according to similarity of meaning. (b) Ordering of modal adjectives according to their degree of qualification.
Results from the lexical negation condition were very similar to the results from the affixal negation condition. The order of the adjectives based on the one-dimensional solutions correlated highly $\left(r_{s}=.93\right.$, $\mathrm{p}<.001$ ); also, the order of the adjectives based on the ordering results correlated highly $\left(\mathrm{r}_{\mathrm{s}}=.99, \mathrm{p}<.001\right)$. (In order to compare the two sets of adjectives, the position of an affixally negated form of an adjective was compared with the position of its corresponding lexically negated form.)

Comparing only the affixally negated forms with their corresponding lexically negated forms, the two types of negation appeared to have similar effects. Comparing the one-dimensional solutions, the order of the affixally negated forms was highly similar to the order of the corresponding lexically negated forms $\left(\mathrm{r}_{s}=.86\right.$, $\mathrm{p}<.05$ ). Comparing the ordering results, the order of the affixally and lexically negated forms was identical $\left(r_{s}=1.00, p<.001\right)$.

Given either affixal or lexical negation, the order of modal adjectives along a dimension is preserved. Based on the ordering data, the order of affirmative adjectives correlated highly both with the order of the corresponding lexically negated adjectives $\left(r_{s}=.98, p<.001\right)$ and with the order of the corresponding affixally negated adjectives $\left(\mathrm{r}_{\mathrm{s}}=.83, \mathrm{p}<.05\right)$.

\section{Discussion}

Asking people to judge the similarity of meaning of modal adjectives and asking them to judge the degree of qualification conveyed by modal adjectives produce highly similar results; this correspondence supports the conclusion that an important aspect of the meaning of modal adjectives is that they can all be used to qualify the truth of a statement. Modal adjectives, then, can be related to each other in terms of a shared conceptual component, qualification. The ordering results, in particular, indicate that the basis for the obtained onedimensional scaling solution is an underlying unidimensional scale of qualification. This kind of semantic representation for modal adjectives is similar to that attributed to quantifiers (Holyoak \& Glass, 1978): Word meanings in both the domains of modal adjectives and of quantifiers appear to be represented in terms of degrees along an internal unidimensional scale.

Results from both the affixal and lexical negation conditions indicate that negated modal adjectives are more qualifying, or weaker, than affirmative modal adjectives. Moreover, the order of negated adjectives is about the same as the order of their corresponding affirmative forms: The order of modal adjectives along the dimension of qualification is preserved under negation, rather than inverted. Both types of negation, affixal and lexical, preserve the order of modal adjectives along the dimension of qualification. Finding that the effects of affixal and lexical negation on the order of affirmative adjectives is the same, however, does not indicate that the two types of negation are equivalent. 


\section{EXPERIMENT 2}

In order to compare affixal and lexical negation of modal adjectives, judgments were elicited when both forms were presented together. In Experiment 2, people performed both sorting and ordering tasks with the same set of sentences: The set included affirmative adjectives, affixally negated adjectives, and lexically negated adjectives. By directly comparing corresponding affixally and lexically negated forms, it is possible to determine whether one type of negation is consistently more qualifying than the other.

In Experiment 1, "certain" and "definite" were, in general, judged to be least qualifying. In the ordering instructions, however, the words "certain" and "certainty" were mentioned. For example, subjects were asked to put sentences in order according to "the certainty of the event." This may have influenced their judgments about the adjective "certain." To avoid mentioning a modal adjective in the instructions, the ordering instructions were changed so that the sentences were ordered according to the degree to which "it will happen" was "true." Thus, in Experiment 2, the ordering instructions were changed and the proportion of negated items was increased in both the sorting and ordering tasks. The sorting and ordering tasks were otherwise identical to those in Experiment 1.

Another way to qualify a statement is to assert its probability of being true (Lyons, 1977). For example, when a reporter says, "There's an $80 \%$ chance that it will rain," the reporter does not express absolute commitment to the truth of "it will rain"; the reporter qualifies the truth of this statement by asserting that the probability of the statement's being true is $80 \%$. The relation between modal adjectives and subjective probability might be expected to be comparable to the relation between quantifiers and subjective quantity. According to Holyoak and Glass (1978), the internal scale for quantifiers closely resembles an analogue to subjective quantity; for modal adjectives, the internal scale of qualification may be an analogue to subjective probability.

To determine whether this relation holds, subjects performed an additional task. For each modal sentence presented in the sorting and ordering tasks, subjects assigned a probability value to the qualified statement "it will happen." An ordering of modal adjectives can be obtained based on this judged probability of the statement "it will happen" in each presented sentence. Recall that the ordering task provides an ordering of modal adjectives based on their degree of qualification. These two orderings can be compared to determine whether the subjective probability of a modally qualified statement is analogous to the degree of qualification conveyed by the modal adjective.

\section{Method}

Subjects. Forty-two adult volunteers were paid $\$ 3 / \mathrm{h}$ for their participation in the experiment. Since one subject did not complete the probability task, his data were eliminated from the analysis of the probability results.

Materials. The stimuli used in each task were sentences of the form "It is __ that it will happen." The same eight affirmative adjectives, lexically negated adjectives, and affixally negated adjectives used in Experiment 1 were substituted into this frame. All 24 sentences, however, were presented in each task; the same 24 sentences were presented in the sorting task, the ordering task, and the probability task. Each of the 24 sentences was typed on a computer punch card for easy duplication.

Procedure. Twenty-four sentences were presented in a different random order for each subject in each task. First, subjects sorted the sentences into groups such that the sentences in any group were similar in meaning. The procedure for the sorting task was identical to that used in Experiment 1. Next, subjects ordered the sentences according to the degree to which "it will happen" was true (i.e., the sentence in which "it will happen" was most true was to be put first, etc.). After completing the ordering task, subjects performed a distractor task: They estimated the frequency of use of each affirmative modal adjective in various contexts. The following day, subjects performed a probability assignment task. Subjects assigned "any number from 0 to 100 , including 0 and 100 , to describe the chances that it will happen, according to each sentence." Thus, an interpolated task and approximately $24 \mathrm{~h}$ intervened between the administration of the ordering task and the probability assignment task.

\section{Results}

The major results for the sorting, ordering, and probability tasks will be presented in order, followed by results specifically regarding negation.

The sorting task yielded a 24 by 24 similarity matrix that was analyzed by the TORSCA multidimensional scaling program (Young \& Torgerson, 1967). A onedimensional solution of low stress was obtained and is shown in Figure 4 on the left (stress $=.1103$ ). The adjectives range from "definite" or "certain" at the top to "not possible" at the bottom.

Based on the ordering data, the median position for each sentence is shown in Figure 4 on the right. The adjectives are arranged from "definite," the least qualified, at the top, to "impossible," the most qualified, at the bottom. The largest interquartile range was 8.47 , but the range usually did not exceed 5.00. Although the sentences were ordered according to how true, rather than how certain, "it will happen" was, the position of the word "certain" is about the same as that in Experiment 1 .

The order of the modal adjectives obtained from the one-dimensional solution corresponds to the order obtained from the ordering results $\left(\mathrm{r}_{\mathrm{s}}=.86, \mathrm{p}<.001\right)$. Despite rewording the instructions in the ordering task and increasing the proportion of negated adjectives in both the sorting and ordering tasks, the results of Experiment 2 were highly similar to the results of Experiment 1.

Figure 5 presents the median probability value assigned to each sentence. The highest median probability value was obtained for the sentence containing "definite"; the lowest value was obtained for the sentence containing "impossible." Many subjects, however, did not assign the extreme value of 100 to "definite" or the extreme 


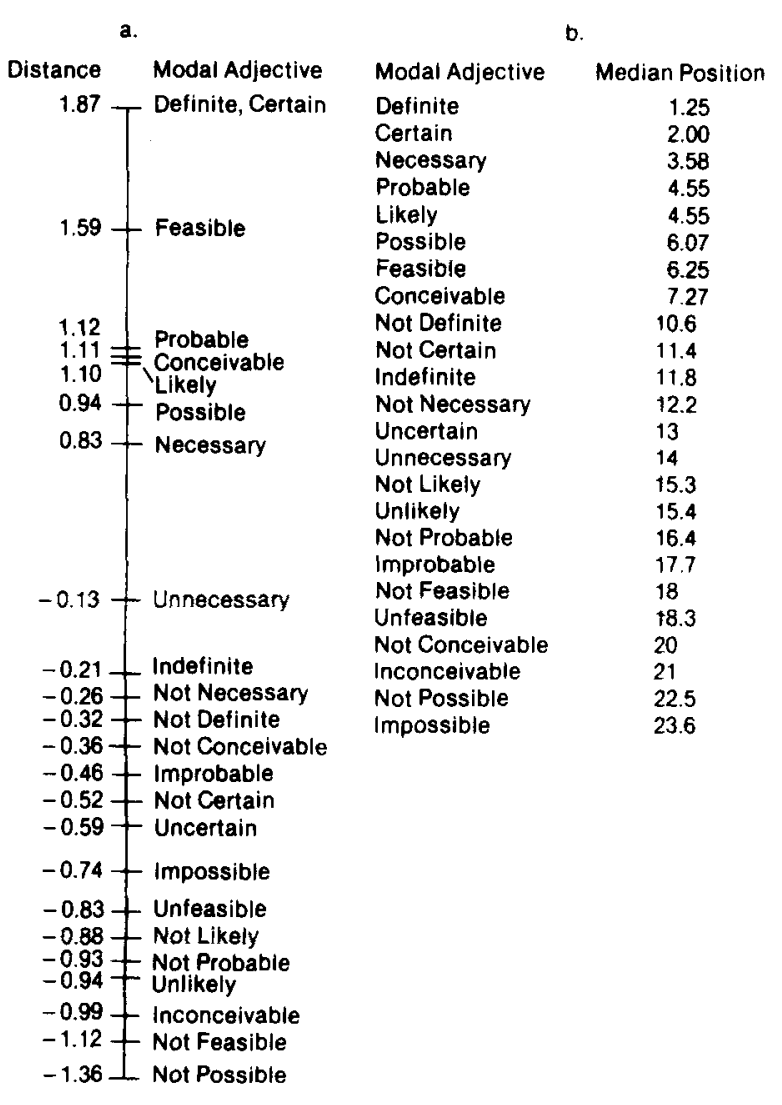

Figure 4. Experiment 2: (a) One-dimensional scaling solution based on sorting modal adjectives according to similarity of meaning, including both affixally and lexically negated forms together. (b) Ordering of modal adjectives according to their degree of qualification, including both affixally and lexically negated forms.

value of 0 to "impossible": Forty-two percent of the subjects did not assign a probability value of 100 to "definite," and 59\% did not assign 0 to "impossible."

The order of modal adjectives based on their median probability values correlated significantly with the results from the sorting and ordering tasks $\left(\mathrm{r}_{\mathrm{s}}=.88\right.$, $\mathrm{p}<.001$, and $\mathrm{r}_{\mathrm{s}}=.99, \mathrm{p}<.001$, respectively). Thus, as might be expected, the correlations reported so far indicate that results from the sorting, ordering, and probability tasks were highly similar.

Since results regarding negation are similar for all three tasks, only the results from the probability task will be addressed in detail. Figure 6 shows the affirmative modal adjectives on the left and the corresponding affixally negated adjectives on the right. The median probability value associated with each adjective is indicated by the point at which the line leading from the adjective touches the diagonal. In other words, the lines leading from the two sets of adjectives show how the affirmative and negative modal adjectives are mapped onto a scale of qualification. The order of the affirmative adjectives was approximately the same as that of the corresponding affixally negated adjectives; the affirmative adjectives ranged from "definite" to "possible," and the negated adjectives ranged from "indefinite" to "impossible" $\left(r_{s}=.93, p<.01\right)$.

Figure 7 shows the results of lexically negating the affirmative adjectives. Using "not" rather than a prefix produced the same pattern of results: The order of the affirmative adjectives was about the same as that of the corresponding lexically negated adjectives $\left(r_{s}=.83\right.$, $\mathrm{p}<.05$ )

Since affixally and lexically negated forms were presented together in all three tasks, it is possible to

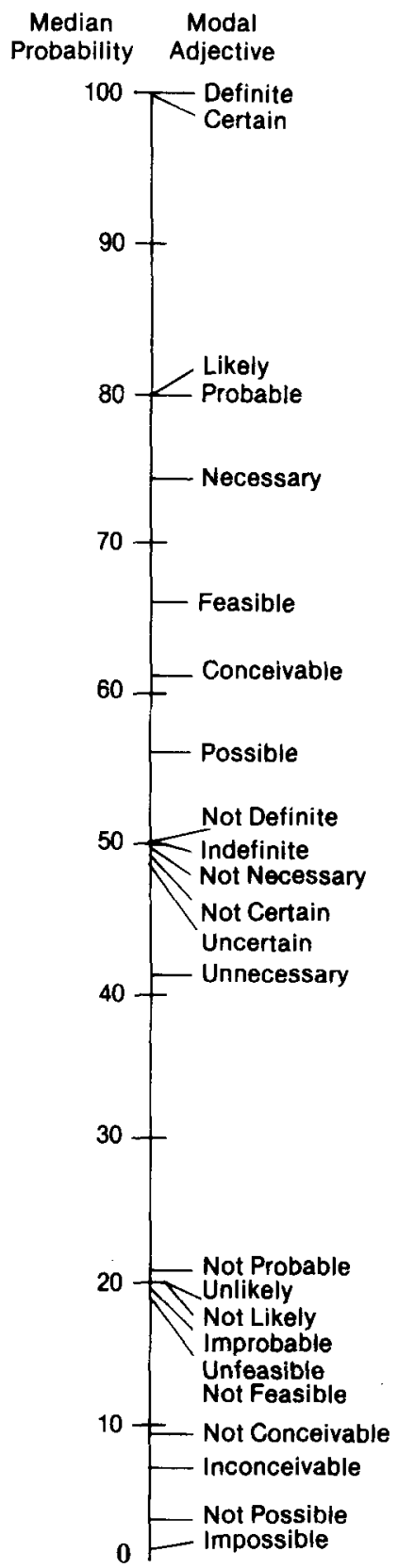

Figure 5. Experiment 2: Median subjective probability values assigned to modal adjectives. 
AFFIRMATIVE

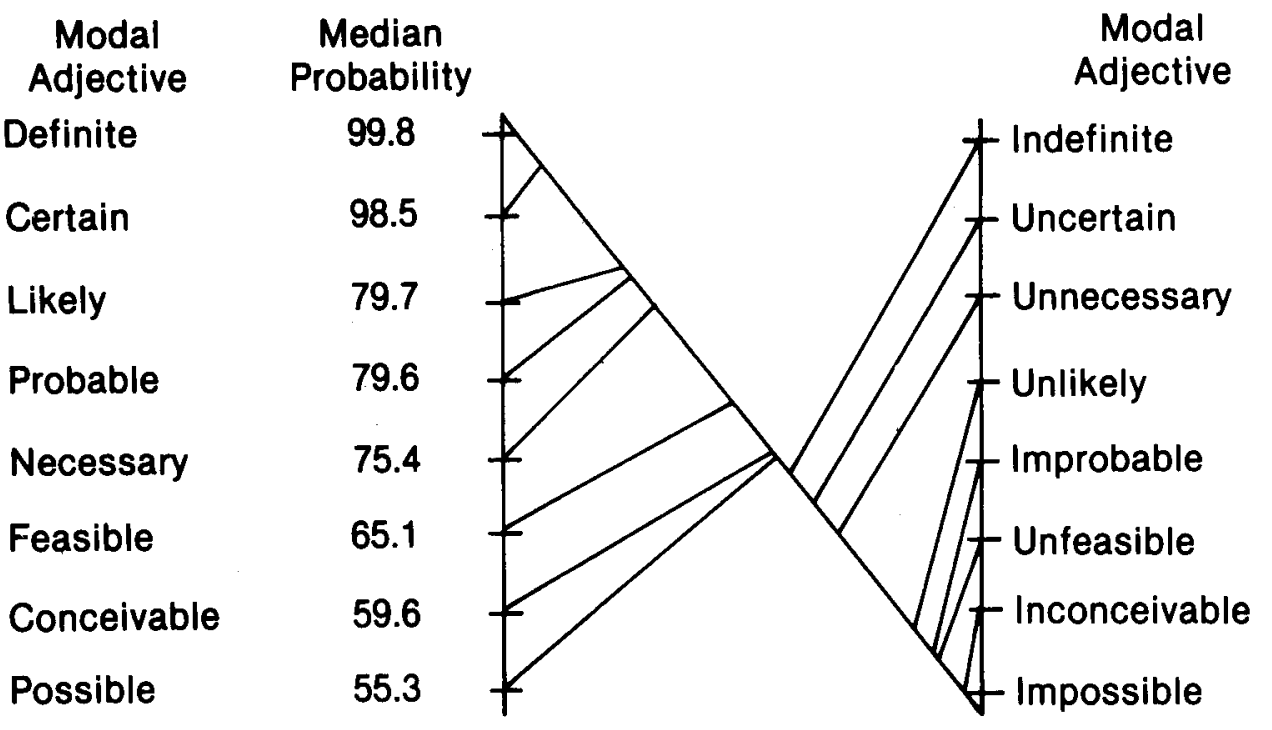

NEGATIVE Median
Probability

50.1

Figure 6. Experiment 2: Affixal negation of modal adjectives.

AFFIRMATIVE

\begin{tabular}{|c|}
\hline $\begin{array}{c}\text { Modal } \\
\text { Adjective }\end{array}$ \\
\hline Definite \\
\hline Certain \\
\hline _ikely \\
\hline Probable \\
\hline \\
\hline Feasible \\
\hline Conceivable \\
\hline Possible \\
\hline
\end{tabular}

\section{Median Probability}

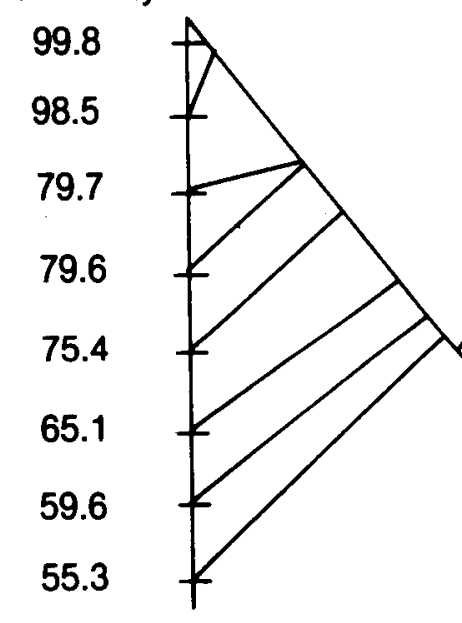

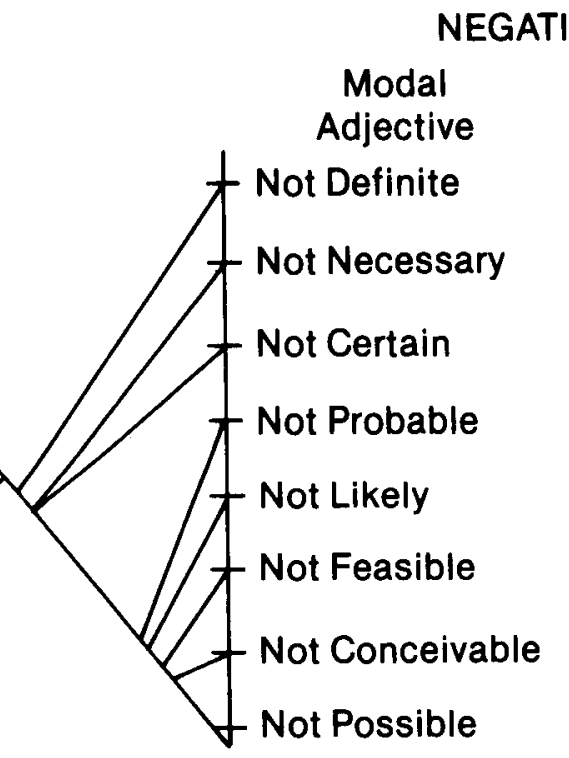

\section{NEGATIVE}

Median Probability

50.3

49.8

49.8

20.6

20.1

19.7

9.7

Figure 7. Experiment 2: Lexical negation of modal adjectives.

compare judgments regarding corresponding affixally and lexically negated forms. Affixally negated forms were consistently judged to be more qualifying than their corresponding lexically negated forms. In other words, comparing such pairs as "inconceivable/not conceivable," "inconceivable" was consistently judged to be more qualifying than "not conceivable," and so on. This relation was reversed for only 1 pair among the 16 pairs in the ordering and probability tasks, and there was one tie.

The slight stress involved in the one-dimensional solution may have been great enough to obscure the consistent but small differences between affixally and lexically negated forms. For this reason, less confidence can be attached to the more equivocal results shown there. Thus, in general, affixal negation was consistently, although slightly, more qualifying than lexical negation.

\section{Discussion}

Experiment 2 replicates the finding from Experiment 1 that judging meaning similarities among modal adjectives corresponds to ordering the adjectives along a dimension of qualification; this correspondence was obtained with different groups of subjects and slightly differing materials and instructions in the two experiments. (These results, nevertheless, do not rule out the 
possibility that dimensions other than qualification might characterize the meanings of modal adjectives in other tasks or contexts.)

The degree of qualification conveyed by modal adjectives also correlated with subjects' assignment of probability values to qualified statements. Although the connection between modal adjectives and subjective probability is apparent for such adjectives as "likely" and "probable," it is not logically required that lexical qualification should so closely resemble an analogue to subjective probability.

The probability task also provided subjects a means of indicating that a statement was absolutely definite or absolutely impossible: Subjects could use the extreme endpoints of the scale, 0 and 100 , to indicate absoluteness. Many subjects, however, did not assign the value of 0 to the word they judged most qualifying or 100 to the word judged least qualifying; subjects often refrained from assigning the most extreme values even to "impossible" and "definite."

As in Experiment 1, negated adjectives were more qualifying than affirmative adjectives and, under negation, the order of adjectives along the dimension of qualification was preserved. Affixal negation, however, differed slightly but consistently from lexical negation: Affixally negated forms were more qualifying, or weaker, than their corresponding lexically negated forms. This result is consistent with Zimmer's (1964) observation that affixal negation is associated with strong negation, whereas negation with "not" is sometimes associated with weak negation.

\section{GENERAL DISCUSSION}

Modal logicians traditionally emphasize absolute distinctions, or discrete contrasts, between such adjectives as "possible" and "necessary." On the other hand, the linguist John Lyons (1977) emphasizes the ordered, continuous nature of modal meanings, that they reflect degrees of qualification rather than absolute states of affairs, and he specifically includes probabilistic adjectives in the modal domain. Results discussed earlier, from sorting and ordering tasks, suggest that modal adjectives are internally represented in terms of degrees along a unidimensional scale of qualification. Results from the probability assignment task, in particular, indicate that even interpretations of such adjectives as "impossible" and "definite" were often not absolute; for example, subjects tended to assign a range of very low, but nonzero, probability values to "impossible."

Although the results obtained in this study are especially congruous with Lyons' (1977) analysis of modal words, I am not claiming that some contexts would not support absolute interpretations for modal words: For the sentence "A bachelor is necessarily unmarried," a logical, or absolute, reading of "necessarily" is salient. On the other hand, in the sentence "An education is necessary in order to get a good job," "necessary" would probably mean "very important, but not compulsory." The present results appear to be most relevant to a description of the usual, ordinary interpretations of modal adjectives, as opposed to their more formal, academic, or technical uses.

Negation was found to operate on modal adjectives by translating them downward along a scale of qualification; this negative translation preserved the order of the corresponding affimative adjectives. If the coordinates along the one-dimensional solution are taken to represent the degree of qualification of the adjectives, it is as if negation operates on an affirmative adjective by subtracting a certain quantity from the affirmative value. Negation is not analogous to a multiplicative process by which the affirmative value is multiplied by -1 . If negation were like a multiplicative process, the order of the negated adjectives would be the inverse of the order of the affirmative adjectives. Instead, the orders are approximately the same.

Hersh and Caramazza (1976) have examined the effect of negation on combinations of "very" with the adjectives "large" and "small." Subjects judged the applicability of each constructed phrase (e.g., "not very small") to the same series of squares of varying sizes. Hersh and Caramazza compared the applicability of corresponding affirmative and negative phrases (e.g., "very small/not very small") across the square sizes. When a phrase's applicability across square sizes was plotted, the function for a negative phrase was the complement of the function for the affirmative phrase. This complement operation is a subtractive process (Zadeh, 1965). Therefore, the operation of negation on a unidimensional continuum of size seems to correspond to its operation on a unidimensional continuum of qualification. Since the semantic structure underlying adjectives that appear to exemplify inversion under negation (e.g., "trustworthy" and "reliable") has not been investigated, the factors specifically controlling the effects of negation are not, at present, identifiable.

The present study compared the effects of two types of negation, affixal and lexical, on modal adjectives. Affixally negated adjectives were found to be consistently more qualifying, more strongly negative, than their lexical counterparts. There are at least two factors that influence whether negation is interpreted strongly or weakly. First, according to Lyons (1977), negation of a "gradable expression" will "always tend" to produce strong, rather than weak, negation, even when a language lexicalizes the strong negation, as in "impossible" (p. 773). Thus, since modal adjectives are gradable expressions, both lexical negation, as in "not possible," and affixal negation, as in "impossible," should produce strong negation. Since lexical negation was consistently weaker than affixal negation, Lyons' observations cannot be extended to this domain of gradable expressions. The strength of negation also seems to depend on the closeness of association between a negative and what is negated (Jespersen, 1929). For example, negation of the proposition "John is a fool" is stronger in "I said that John is not a fool" than in "I did not say that 
John is a fool." Although, in the sentences used in this study, both the affixal and lexical negative elements are adjacent to the adjective being negated, the detached character of lexical negation seems sufficient to indicate weaker negation.

\section{REFERENCES}

Clark, H. Semantics and comprehension. In T. A. Seboek (Ed.), Current trends in linguistics (Vol. 12): Linguistics and adjacent arts and sciences. The Hague: Mouton, 1972.

Clark, H., \& Clark, E. Psychology and language: An introduction to psycholinguistics. New York: Harcourt Brace Jovanovich, 1977.

Chiff, N. Adverbs as multipliers. Psychological Review, 1959, $66,27-44$.

Falmagne, R. J. Reasoning: Representation and process. Hillsdale, N.J: Erlbaum, 1975.

Hersh, H., \& Caramazza, A. A fuzzy set approach to modifiers and vagueness in natural language. Journal of Experimental Psychology: General, 1976, 105, 254-276.

HolyonK, K., \& Glass, A. Recognition confusions among quantifiers. Journal of Verbal Learning and Verbal Behavior, 1978, 17, 249-264.

HolyoAK, K., \& WALker, J. Subjective magnitude information in semantic orderings. Journal of Verbal Learning and Verbal Behavior, 1976, 15, 287-299.

Hughes, G., \& CResswell, M. An introduction to modal logic. London: Methuen, 1974.
Jespensen, O. The philosophy of grammar. London: Allen \& Unwin, 1929.

LeEch, G. Semantics. Harmondsworth: Penguin, 1974.

Lyons, J. Semantics (Vol. 2). Cambridge, England: Cambridge University Press, 1977.

MiLLER, G. A. Empirical methods in the study of semantics. In D. D. Steinberg \& L. A. Jakobovits (Eds.), Semantics: An interdisciplinary reader in philosophy, linguistics, and psychology. Cambridge, England: Cambridge University Press, 1971.

Miller, G. A., \& Johnson-Laird, P. N. Language and perception. Cambridge, Mass: Harvard University Press, 1976.

REvurs, R. Syllogistic reasoning: Logical decisions from a complex data base. In R. J. Falmagne (Ed.), Reasoning: Representation and process. Hillsdale, N.J: Erlbaum, 1975.

Standard College Dictionary. New York: Funk and Wagnall's Harper and Row, 1977.

Taplin, J., \& Staudenmayer, H. Interpretation of abstract conditional sentences in deductive reasoning. Journal of Verbal Learning and Verbal Behavior, 1973, 12, 530-542.

Youna, F., \& Togenson, R. Torsca. A Fortran IV program for Kruskal-Shepard multidimensional scaling. Behavioral Science, 1967,

ZADEH, L. Fuzzy sets. Information and Control, 1965, 8, 338-353.

Zimmer, K. Affixal negation in English and other languages: An investigation of restricted productivity. Supplement to Word, 1964, 20(Whole No. 5), 1-105.

(Received for publication July 28, 1980; revision accepted April 6, 1981.) 